\title{
Toxicología del cadmio. Conceptos actuales para evaluar exposición ambiental u ocupacional con indicadores biológicos
}

\author{
AUGUSTO RAM ÍREZ \\ M édico del Trabajo. American College of $O$ ccupational and Environmental M edicine.
}

\begin{abstract}
RESUMEN
Desde el inicio de la Era Industrial, y especialmente después de la II Guerra M undial, el hombre vive expuesto a cada vez más contaminantes y ha enfrentado grandes desastres ambientales, como M inamata, Bophal y Chernovyl. El cadmio es un tóxico que produce contaminación ambiental e industrial y, en el ser humano, causa al teraciones a nivel enzimático, renal, respiratorio y digestivo, con el agravante de tener una vida media muy prolongada. Para enfrentar el problema deberíamos hacer un inventario de las principales fuentes de contaminación industrial, en metalurgia principalmente, para fiscalizarlas. De otro lado, es necesario proteger al trabajador, aplicando los criterios de valores límites umbrales (TLVs) e indicadores biológicos de exposición (BEIs), acordes con el actual estado del conocimiento.
\end{abstract}

Palabras clave: Cadmio; envenenamiento por cadmio; exposición a riesgos ambientales; contaminación ambiental; indicadores de contaminación.

\begin{abstract}
CADMIUM TOXICOLOGY. CURRENT CONCEPTS TO DETERMINE ENVIRONMENTAL OR OCCUPATIONAL EXPOSITION BY MEANS OF BIOLOGICAL INDICATORS SUM MARY

With the beginning of the Industrial Era, specially after the II World War, man lives exposed progressively to more pollutants and has confronted huge environmental disasters like M inamata, Bophal, and Chernovyl. Cadmium is an environmental and industrial toxic pollutant that produces in man effects at the enzymatic, renal, respiratory and digestive levels, aggravated by its very extended mean life. To face this problem, we would have to make a survey of the principal sources of industrial pollution, mainly metallurgic, in order to protect the worker, applying TLV $s$ and BE Is criteria concurrent with the state of the art.
\end{abstract}

Key words: Cadmium; cadmium poisoning; environmental exposure; environmental pollution; pollution indicators.

\section{INTRODUCCIÓN}

El hombre produce constantemente desechos industriales, que son expelidos directamente a la atmósfera, inmenso mar que nos rodea y en cuyo fondo vivimos. A costumbrado a vaciar estos desechos en cursos de agua y mares que parecían

Correspondencia:

Augusto V. Ramírez

Las M andarinas 210 Ap. 306

Lima 12, Perú

E-mail: augustovram@yahoo.es inagotables e insensibles, ahora sabe que, con el aumento de la población y el acmé de la Era Industrial, si quiere seguir disponiendo de agua y aire limpios debe controlar los desechos que genera y arroja sin contemplación alguna. $\mathrm{Ha}$ iniciado entonces un acto de contrición tendiente a hacer de nuestro planeta un lugar saludable. Sin agua, el hombre puede vivir horas y aún días, pero sin aire muere a los 8 ó 10 minutos; de ahí que tan o más vital que el agua sea el aire. Nuestro medio ambiente está contaminado, además, de deshechos humanos, por basura, radiación, ruido, químicos de la más insospechada natura- 
leza, como plaguicidas, pesticidas y gases, que ha creado el hombre para su comodidad o para matarse entre sí; o vapores, humos y polvos provenientes de disímiles fuentes, como combustibles fósiles de vehículos y actividad industrial de fundiciones y refinerías, todos en megacantidades que interfieren la salud y bienestar humano y el del planeta, y que en cualquier momento desencadenan fenómenos catastróficos de morbimortalidad, tal como ocurrión en L ondres, Donora, Chernovyl, Bophal, Seveso $\left({ }^{1}\right)$ o, el más reciente, en Baia $M$ are.

\section{EI cadmio}

U no de los mayores agentes tóxicos asociado a contaminación ambiental e industrial es el cadmio, pues reúne cuatro de las características más temidas de un tóxico:

1. Efectos adversos para el hombre y el medio ambiente.

2. Bioacumulación.

3. Persistencia en el medio ambiente.

4. "Viaja" grandes distancias con el viento y en los cursos de agua.

El cadmio ( $\mathrm{C}$ d, número atómico 48 , masa atómica 111,40$)$ se obtiene como subproducto del tratamiento metalúrgico del zinc y del plomo, a partir de sulfuro de cadmio; en el proceso hay formación de óxido de cadmio, compuesto muy tóxico. Además de contaminar el ambiente desde su fundición y refinación, contamina también por sus múltiples aplicaciones industriales.

Los principales usos y aplicaciones del cadmio o sus compuestos son:

- Como pigmento en pinturas, esmaltes, plásticos, textiles, vidrios, tintas de impresión, caucho, lacas, etc.

- En aleación con cobre, aluminio y plata.

- En la producción de pilas de cadmio-níquel.

- Como estabilizador de termoplásticos, como el PVC.

- En fotografía, litografía y procesos de grabado.
- Como "endurecedor" de ruedas y llantas de automóvil.

- En fabricación de foto - conductores y células solares fotoeléctricas.

- El electroplatinado.

- En fabricación de "controles" de reactores nucleares.

U sos tan diversos y su larga vida media no permiten el reciclaje, por lo que se acumula progresivamente en el ambiente.

En lo ambiental, el cadmio es un elemento relativamente raro en la litosfera. Por afinidad química, se le encuentra junto al zinc, en proporción muy variable. $L$ as principales fuentes de contaminación son: la minero metalurgia de metales no ferrosos, la metalurgia del hierro y acero, la fabricación de fertilizantes fosfatados, la incineración de residuos de madera, carbón o "plásticos", la combustión de aceite y gasolina y las aplicaciones industriales de cadmio. La concentración de cadmio en aire de áreas industriales varía de 9,1 a $26,7 \mu \mathrm{g} / \mathrm{m}^{3}$ frente a 0,1 a $6 \mathrm{ng} /$ $\mathrm{m}^{3}$ en el aire de áreas rurales. El tiempo de permanencia del cadmio en suelos es de hasta 300 años y el $90 \%$ permanece sin transformarse $\left.{ }^{(}\right)$. El cadmio llega al suelo de los terrenos agrícolas por deposición aérea $(41 \%)$, con los fertilizantes fosfatados $(54 \%)$, por aplicación de abono de estiércol $(5 \%)$ y, en el Perú, frecuentemente por efluentes que contienen residuos líquidos y sólidos de plantas hidrometalúrgicas de cadmio. $\mathrm{H}$ emos encontrado que, en suelos contaminados, los niveles de cadmio al canzan val ores de hasta 1 $\mathrm{mg}$ de $\mathrm{Cd} / \mathrm{g}$ y el agua de ríos contaminados puede contener hasta $0,14 \mathrm{mg}$ de $\mathrm{Cd}$ por $\mathrm{L}(2,3)$.

De menor importancia en contaminación ambiental por cadmio son las partículas de polvo de caucho provenientes de las ruedas de automóvil, de la galvanización con zinc de tuberías, de plásticos que contienen pigmentos coloreados de cadmio y de la industria de cerámica glaseada. Se ha descrito intoxicación por uso doméstico de algunos utensilios bañados en cadmio o por usar cubiertos de plata al eada con cadmio y pinturas a base de cadmio. En no 
expuestos ocupacionalmente, la alimentación y el tabaco constituyen las dos fuentes principales de exposición. Los fumadores pueden absorber cantidades comparables a la ingestión diaria normal con la dieta (IDD), es decir, inhalan 0,1 a $0,2 \mu \mathrm{g}$ de $\mathrm{Cd}$ por cigarrillo, con una absorción aproximada del $50 \%$. Por esto, los fumadores deben forman un grupo especial, cuando se investiga exposición a cadmio $(4,5)$.

El cadmio entra en la alimentación humana con los vegetales y productos animales. Se fija a las plantas más rápidamente que el plomo. Los frutos y semillas contienen menos cadmio que las hojas. El pescado, los crustáceos, el riñón e hígado de animales acumulan cadmio en grado relativamente elevado. La IDD se estima entre 10 y $85 \mu \mathrm{g}$. A lgunos países, como Japón y Canadá, tienen dietas con valores de C $\mathrm{d}$ más elevados. Organizaciones internacionales han propuesto como límite tolerable medio en Ia IDD $70 \mu \mathrm{g} /$ semana. El agua potable contribuye en menor grado, pues debe contener un máximo de $1 \mu \mathrm{g} \mathrm{Cd} / \mathrm{L}\left({ }^{6}\right)$.

En cuanto a la exposición industrial, el cadmio ingresa por inhalación de humos y vapores o por ingesta de los polvos de óxido de cadmio de sus compuestos. Los puestos de trabajo más agresivos en exposición laboral se haIlan en la industria metalúrgica primaria de cadmio y de plomo-zinc, en la producción y uso de pigmentos de cadmio, en la producción de estabilizadores de plásticos con cadmio y en la fabricación de baterías de cadmio-níquel. En el hombre, los efectos por exposición a cadmio están bien documentados: es irritante y tóxico respiratorio, tóxico renal, causa cierto tipo de osteomalacia y se le ha asociado con cáncer $\left({ }^{7}\right)$.

\section{Toxicocinética}

La absorción, distribución y excreción del cadmio en el ser humano en condiciones "normales" de exposición ambiental, las mostramos sintetizadas en la Figura 1. El contenido corporal de cadmio se incrementa con la edad hasta los 50 años. E n los adultos, la carga corporal de cadmio puede llegar a 40 miligramos, depen- diendo de la situación geográfica y sobretodo del hábito de fumar, pues en un fumador la carga alcanza el doble ( $\left.{ }^{8}\right)$.

En condiciones "normales" de distribución, el cadmio absorbido se excreta principalmente por orina y en menor cantidad con la bilis, aunque pequeñas porciones puedan eliminarse con sudor, pelo y aún secreción gastrointestinal, pero el $C d$ que sale con heces en su mayor parte es el que no se absorbió $\left({ }^{9}\right)$.

En exposición no laboral, la alimentación es la fuente más importante de ingesta de cadmio. $\mathrm{L}$ a absorción por el tracto gastrointestinal es de aproximadamente $50 \%$. La dieta deficiente en Ca, Fe o proteína incrementa la velocidad de su absorción.

En sangre encontramos aproximadamente $0,06 \%$ del contenido corporal de $\mathrm{Cd}$ y más del $50 \%$ está en los hematíes unido inestablemente a una seudoproteína, la metalotioneína. La metalotioneína es el "medio de transporte" del cadmio en el plasma sanguíneo. El aclaramiento sanguíneo del $\mathrm{Cd}$ es rápido, se acumula principalmente en el riñón y en adultos no expuestos Ilega a valores entre 7,4 y $8,8 \mathrm{mg}$, lo que representa entre $30 \%$ y $50 \%$ de su contenido corporal. La concentración en la corteza renal es 1,5 veces mayor que la del riñón total y se fija en las células del túbulo proximal. El hígado de adultos no expuestos tiene en promedio $2,7 \mathrm{mg}$ de cadmio.

$\mathrm{L}$ a acumulación de $\mathrm{C} d$ en riñón e hígado depende de la intensidad, del tiempo de exposición y del estado óptimo de la función de excreción renal. En ambos casos se ha encontrado incremento con la edad. Después de sobreexposición alcanza concentraciones el evadas en el hígado; pero con el tiempo el metal se localiza en el riñón. Se ha descrito también que las concentraciones renales de zinc se incrementan al aumentar las de cadmio y que la capacidad de almacenamiento de la corteza es limitada a $300 \mu \mathrm{g} / \mathrm{g}\left({ }^{10}\right)$.

En las células, el cadmio se une a la metalotioneína, proteína cuyo peso molecular es de 6945 u (7 000 dalton) y que contiene 26 gru- 


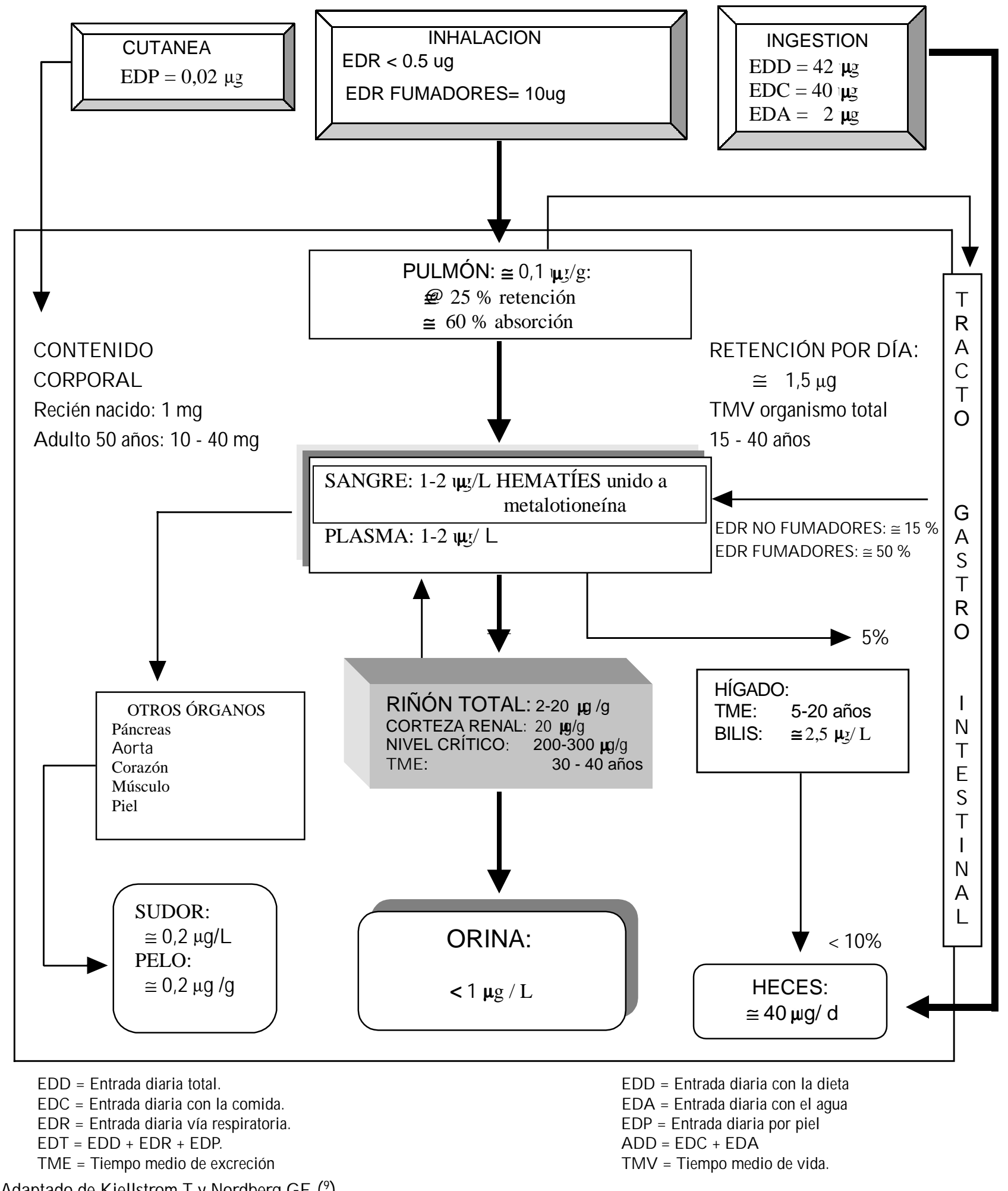

Adaptado de Kjell stromT y Nordberg GF ( ${ }^{9}$ ).

Figura 1. Toxicología del cadmio. Toxicocinética. 
pos SH libres por molécula, debido a la gran proporción de residuos de cisteína. La función principal de esta microproteína es la protección del sistema enzimático celular, aunque se le ha descrito otra función, cual es la de unirse específicamente al cadmio y a otros metales pesados. Su síntesis en hígado, riñón e intestinos es inducida por el cadmio y se conoce por estudios experimentales que el complejo cadmiometalotioneína es más tóxico para los túbulos renales que el cadmio per se. Paradójicamente, cuando la metalotioneína se sintetiza en las células, las protege de la toxicidad del cadmio, pues inactiva el metal. Se ha demostrado también escasa capacidad del riñón para sintetizarla, lo que lo hace insuficiente para fijar el cadmio y da lugar a aparición de las manifestaciones tóxicas ( $\left.{ }^{11}\right)$.

Poblaciones adultas del medio urbano pueden retener hasta $1,77 \mu \mathrm{g} C \mathrm{~d} /$ día; así, en personas de 50 años hay cantidades acumuladas de hasta $32 \mathrm{mg}$; de ellos, la corteza renal contiene aproximadamente $50 \mu \mathrm{g} \mathrm{Cd} / \mathrm{g}$, en un rango que varía entre 15 y 85 . Se sabe, por otro lado, que la concentración de cadmio en el hígado depende del daño de la función renal inducida por el metal, que disminuye la reabsorción del cadmiometal otioneína e incrementa su excreción urinaria. Sólo entonces la concentración de cadmio en hígado excede la de nivel renal ${ }^{12}$ ).

El cadmio atraviesa la barrera placentaria fácilmente, induciendo allí la síntesis de metalotioneína, con la que forma el complejo cadmio-metalotioneína, que se acumula progresivamente en la placenta durante el embarazo, actuando como mecanismo protector frente al transporte de cadmio al feto. Al término del embarazo, la concentración de cadmio en la placenta es aproximadamente 10 veces más que en la sangre materna. Por el contrario, la concentración de cadmio en el cordón umbilical es alrededor de 2 a 3 veces más baja que en la sangre materna. Por ello, se infiere que el cadmio puede interferir la evolución del embarazo por acción directa sobre el metabolismo de la placenta, pero no por acción directa sobre el feto. En el recién nacido el cadmio sanguíneo es de 30 a $50 \%$ menor que el cadmio en la san- gre materna. La leche materna sólo secreta pequeñas cantidades $\left({ }^{13}\right)$.

En exposiciones laborales, la inhalación es la ruta principal de ingreso y la absorción a partir de esta vía depende del tipo de compuesto inhalado, del tamaño de las partículas y de su retención en el pulmón. El depósito en el pulmón de partículas menores de $5 \mathrm{~mm}$ de diámetro es del orden del $25 \%$ y de ellas aproximadamente el $60 \%$ pasa a la sangre. En grandes fumadores se ha encontrado valores adicionales de absorción por inhalación de hasta el 50\%. L as partículas de cadmio depositadas en la nasofaringe, tráquea y bronquios son transportadas por mecanismo mucociliar a la faringe, desde donde son parcialmente ingeridas. $L$ a absorción percutánea ocurre solamente por contacto con los compuestos orgánicos del cadmio $\left({ }^{1,9}\right)$.

En trabajadores recién expuestos el $\mathrm{Cd}$, aumenta en sangre sólo los primeros 6 meses y luego sus niveles son proporcionales a la concentración en el ambiente laboral. En expuestos ocupacionales, el cadmio se encuentra también en páncreas, pulmón, aorta, corazón y músculos. Elevadas concentraciones en un tejido infieren concentración alta en otros, lo que nos lleva a afirmar que la distribución se determina sólo por el nivel ambiental de $\mathrm{C} d$ y no por alguna función especial $\left.{ }^{13}\right)$.

\section{EI modelo toxicocinético}

La Figura 2 muestra el modelo toxicocinético del cadmio, elaborado a partir de investigaciones en animales y por observaciones clínicas en expuestos $\left({ }^{9}\right)$, que podemos resumirlo así:

Absorción: Es relativamente lenta, con un promedio de 14 días en exposiciones prolongadas. Esto puede no ser importante cuando se calcula concentración de cadmio en órganos de trabajadores 0 individuos expuestos crónicamente, pero sí influencia los cálculos para períodos cortos de exposición.

Vías de ingreso y distribución: Como ya hemos visto en exposición ocupacional, el cadmio procede de dos vías de ingreso: inhalación e 
ingestión. La fracción que pasa a sangre se distribuye en 3 compartimentos de recambio:

- Compartimento 1 de "recambio rápido" y, por tanto, no genera acumulación.

- Compartimento 2 de "recambio medio", constituido por los hematíes, en los que se acumula en pequeñas cantidades.

- Compartimento 3 de "recambio lento"; aquí, una fracción significativa de cadmio se une a la metalotioneína y va a depositarse en los órganos blanco.
Los compartimentos 1 y 3 son los de mayor intercambio con los demás órganos y se estima que desde el compartimento 1 la tasa de transferencia a otros tejidos es de $50 \%$ y al hígado de $16 \%$. Entre los tres compartimentos hay equilibrio dinámico, sin embargo, existiría un aporte extra del riñón hacia el compartimento 1. En el riñón, el compartimento 3, aunque en forma lenta, es el de mayor intercambio y por tanto no se produce acumulación, pero sí filtración por el glomérulo. Una parte se reabsorbe en el túbulo, contribuyendo así a su acumulación. EI excedente se excreta con la orina $\left({ }^{14}\right)$.

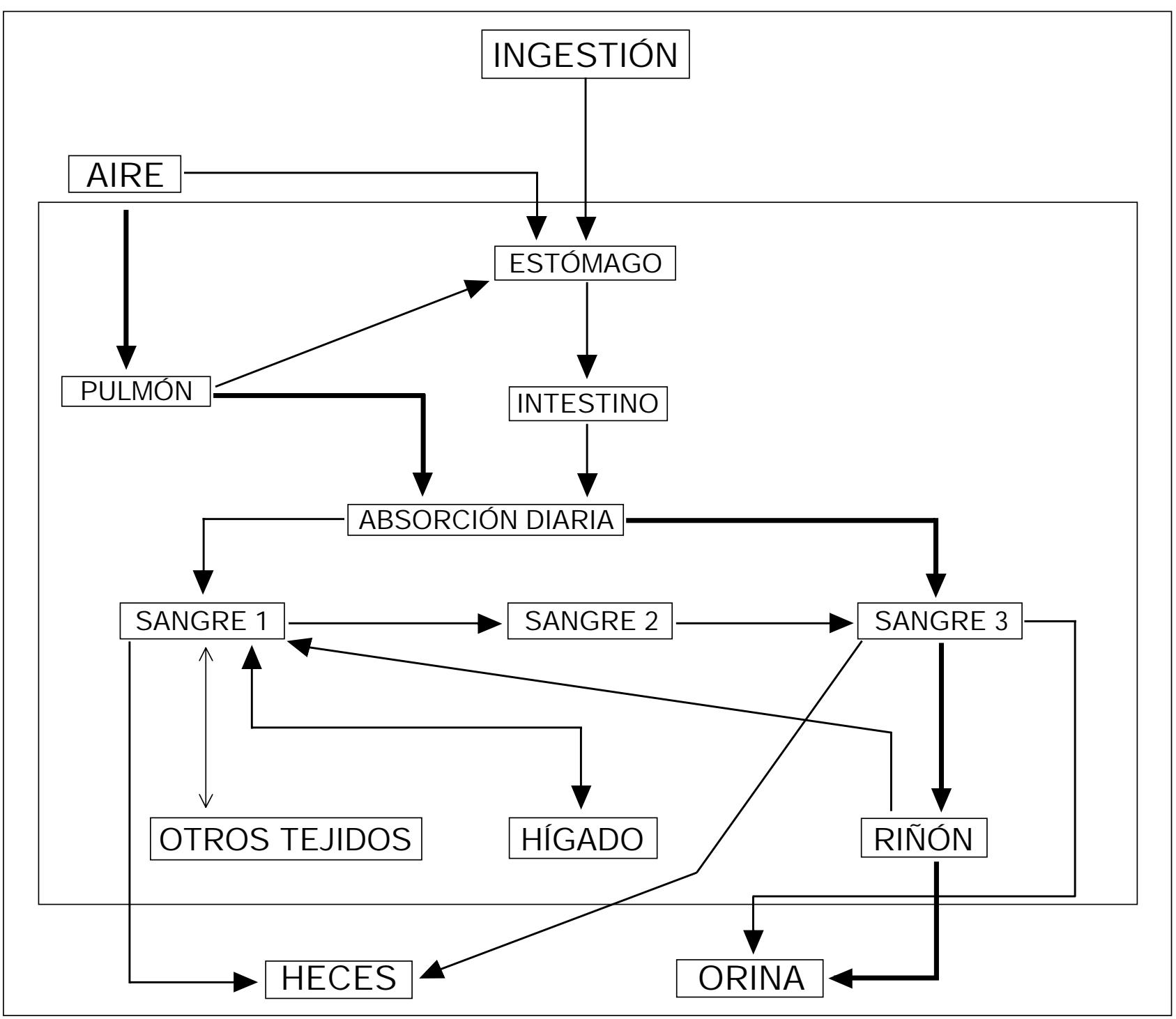

Figura 2. Toxicología del cadmio. Esquema toxicocinético. 
La vida media del cadmio en el organismo total es de 30 a 40 años.

Excreción: $L$ as principales vías de excreción son orina y heces. Por orina, diariamente se elimina $0,007 \%$ del contenido corporal y por heces $0,03 \%$. La vida media de excreción urinaria es de hasta 40 años. Tan sólo una pequeña fracción del cadmio del compartimento sanguíneo y otra del hígado, a través de la vía biliar, se elimina por heces $(9,14)$.

\section{Toxicodinámica}

El cadmio es un xenobiótico y, por tanto, un metal tóxico y no esencial para el organismo, que se acumula en los tejidos humanos. Los órganos blanco son riñón y pulmón. En exposición laboral o ambiental, sus principales efectos tóxicos son: neumonitis química, disfunción renal con proteinuria y microproteinuria y enfisema.

El riñón es más sensible al cadmio que pulmón e hígado y el epitelio del túbulo renal proximal es el punto blanco. Su deterioro se pone de manifiesto por el incremento de proteínas de peso molecular bajo, lo que causa "proteinuria de peso molecular bajo". Concomitantemente, hay alteración de la filtración glomerular, por cambios en la restricción electrostática para la filtración de las proteínas polianiónicas, lo que disminuye su reabsorción y conduce a incrementar la excreción urinaria de proteínas de peso molecular alto, que origina "proteinuria de peso molecular alto". Teóricamente, pues no se ha demostrado in vivo, la acción tóxica del cadmio se debería a su afinidad por radicales de los grupos $-\mathrm{SH},-\mathrm{OH}$, carboxilo, fosfatil, cisteinil e histidil y a su acción competitiva con otros elementos funcionalmente esenciales, $\mathrm{Zn}, \mathrm{Cu}, \mathrm{Fe}$ y $\mathrm{C}$. Sus principales interacciones serían:

- Unión fuerte del Cd a los grupos -SH de las proteínas intracelulares, que inhibiría a las enzimas que poseen estos grupos y

- Desplazamiento del Zn de los enlaces -S- y la consiguiente al teración enzimática y de sus procesos bioquímicos, que se refleja en su deficiencia relativa $\left({ }^{7,14}\right)$.

\section{L as metalotioneínas}

De las metalotioneínas, se sabe ahora que existen dos tipos, que se comportan de forma distinta respecto a acumulación del xenobiótico y a su excreción urinaria. L a fracción de cadmio en plasma se encuentra unida en forma inestable a la metalotioneína 1 y es la que se transfiere rápidamente al riñón. En el tejido renal, en cambio, el cadmio acumulado se encuentra unido en forma relativamente estable a la metal otioneína 2 y su vida media se estima hasta en 68 años. En el hígado, la mayor cantidad de cadmio acumulado se encuentra unido también a la metalotioneína 2 , con una vida media estimada hasta en 19 años. La vida media en sangre es aproximadamente de 2,5 meses. No tenemos datos sobre vida media en otros órganos o tejidos; sin embargo, puede afirmarse que $50 \%$ del contenido total corporal de cadmio está en riñones, hígado y sangre, por lo que a estos 3 órganos se les denomina compartimiento de depósito $(11,15)$.

\section{La beta 2 microglobulina (B 2M)}

La B2M es una proteína de peso molecular bajo, 11707 u (11 800 dalton), de tipo globular sin carbohidratos, compuesta por 100 aminoácidos con un puente - S - S- entre el aminoácido cistina de las posiciones 25 y 81 , con secuencias de aminoácidos y estructura tridimensional muy similar a la de las cadenas cortas y largas de las inmunoglobinas; incluso se les ha establecido un desarrollo genético común. Se le aísla de la orina de intoxicados crónicos con cadmio, pero este aumento no es patognomónico, ya que también se le ha aislado de antígenos humanos histocompatibles y del suero de enfermos con procesos malignos inflamatorios. La B2M es producida constantemente y se elimina casi exclusivamente por vía renal (Figura 3). En sujetos normales se encuentra en baja concentración. Por su tamaño se cree que es filtrada libremente por el glomérulo. Considerando que su concentración en el suero normal es de $2 \mathrm{mg} / \mathrm{L}$ y que su velocidad de filtración glomerular es de $140 \mathrm{~L} /$ día, en los túbulos cada 24 horas hay $280 \mathrm{mg}$ de B 2M $\left({ }^{16,17}\right)$. 
Por otro lado, se ha medido su velocidad de síntesis, usando I $\mathrm{I}^{125}$ - B2M. Se obtuvo una media de $95 \mu \mathrm{g} / \mathrm{hora} / \mathrm{kg}$ y se ha calculado que cada día se liberan entre 150 y 200 mg de B2M . D ebido a su pequeño tamaño, difunde libremente entre los espacios intra y extra vasculares, pero no pasa al espacio intracelular. Estas características moleculares hacen que su catabolismo se regule por filtración glomerular, pasando libremente a través de la membrana glomerular. U na vez filtrada es reabsorbida por células del túbulo proximal ${ }^{\left({ }^{18}\right)}$.

L a Figura 3 presenta una síntesis esquemática de su vía metabólica.

$L$ a concentración urinaria de B2M en el suero del adulto sano depende de edad y sexo, alcanza concentraciones hasta de $1500 \mu \mathrm{g} / \mathrm{g}$ creatinina. La cantidad de B $2 \mathrm{M}$ en los compartimentos intra y extra vasculares puede llegar hasta $8 \mathrm{mg}$. Se le ha calculado una vida media entre 30 y 60 minutos, lo que concuerda con estudios de su metabolismo, que muestran un tiempo de vida promedio de 40 minutos. Su catabolismo se da por filtración glomerular y reabsorción por micropinocitosis a nivel del túbulo proximal, donde se degrada. $L$ a velocidad de desaparición de la B 2 M del suero depende de la velocidad de filtración glomerular, pero no de la función tubular; por tanto, el incremento en la excreción urinaria refleja una falla temprana de la reabsorción tubular. En jóvenes no expuestos, la excreción urinaria es menor a $150 \mu \mathrm{g} / 24 \mathrm{~h}$; pero, en adultos llega a 250. En cuanto a la filtración glomerular, el rango está entre 180 y $275 \mu \mathrm{g} / 24 \mathrm{~h}$, con un coeficiente de reabsorción aproximado de 99,9\%. Por tanto, podemos afirmar que su vida media es muy corta, pues la totalidad de B2M en el intersticio se renovaría cada 2 horas $\left({ }^{18}\right)$.

\section{Evaluación de la exposición con indicadores biológicos}

Para evaluar la exposición a $\mathrm{Cd}$, independiente de la vía ingreso, se debe hacer el muestreo biológico regular con los siguientes indicadores biológicos de exposición (BEIs):
A) cadmio en orina. Cuando el nivel crítico de cadmio en la corteza del riñón aún no se ha alcanzado, este indicador es un buen reflejo de la carga corporal del metal. Pero cuando se aproxima al nivel crítico, refleja más una exposición reciente y al saturarse todos los tejidos con cadmio sus niveles en orina varían entre 5 y 10 $\mu \mathrm{g} / \mathrm{g}$ de creatinina. La American Conference of Governmental Industrial Hygienists (ACGIH) fija como BEI para el período 1997-1998, $5 \mu \mathrm{g}$ $\mathrm{Cd} / \mathrm{g}$ de creatinina $\left({ }^{19}\right)$.

B) Cadmio en sangre. Este BEI refleja exposición muy reciente, de 45 a 60 días, por lo que es un buen indicador laboral cuando se valora mejoras higiénicas o tecnológicas del ambiente de trabajo. Pero, para exposiciones ambientales mínimas, es un parámetro menos satisfactorio que el cadmio en orina. El límite biológico que fija la ACGIH para el período 1997- 98 es de 5 $\mu \mathrm{g} / \mathrm{L}\left({ }^{19}\right)$.

C) Cadmio en heces. Sólo refleja la entrada diaria de cadmio con la dieta (EDD). Por tanto, el $C d$ excretado por esta vía no es útil en exposición laboral ( 9 ).

D) Cadmio en la raíz del pelo. Es un parámetro muy variable cuando valora diferentes niveles de exposición, pero refleja más adecuadamente exposiciones ambientales. No se ha establecido su relación con niveles de cadmio en riñón o hígado. Tampoco nos sirve para diferenciar si lo que expresa es $\mathrm{Cd}$ ambiental o simplemente se libera a partir del $\mathrm{Cd}$ atesorado por el organismo. Por esta razón, no se le utiliza como indicador de exposición laboral $\left({ }^{10}\right)$.

\section{Evaluación de la acción toxicodinámica}

Si se tiene certeza de exposición, medida por la carga corporal de cadmio, pero se quiere conocer el daño que el xenobiótico pudiera haber causado a riñón, hígado o pulmón, usaremos los siguientes indicadores:

- Proteínas totales en orina de 24 horas.

En exposición a cadmio proteínas urinarias mayores a $1 \mathrm{~g}$ en 24 horas indican daño re- 


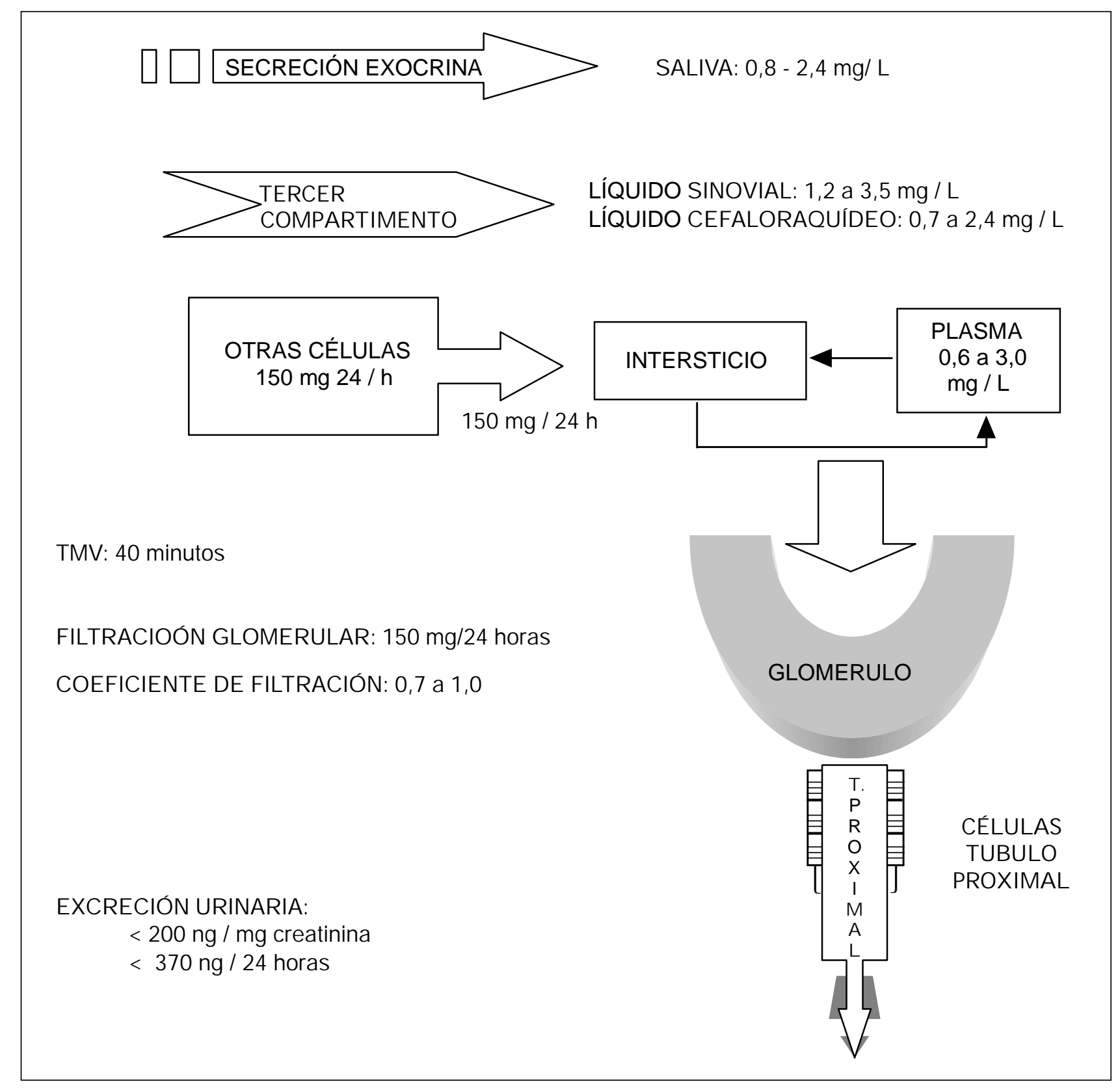

Figura 3. Toxicología del cadmio. Vía metabólica de la beta 2 microgl obulina.

nal; pero ya desde $0,2 \mathrm{~g} / \mathrm{L}$ se les considera críticas.

- Proteinuria de peso molecular bajo:

B 2M , lisozima $\beta$ galactosidasa, $\pi$ glutation -S- transferasa ( $\pi$ GST) y ribonucleasa metalotioneína.
A unque este grupo de proteínas de peso molecular bajo y enzimas, individualmente en orina no son específicas para evaluar exposición ocupacional o ambiental, son índices ampliamente usados para valorar el grado de disfunción renal tubular causado por la 
exposición de $\mathrm{Cd}$. El inconveniente de la B $2 \mathrm{M}$ es que se degrada en vejiga y, con el incremento de la edad, aumenta su cantidad en la orina; así tenemos que en mayores de 50 años se halla cantidades "normales" de hasta 1,8 $\mathrm{mg} / \mathrm{g}$ de creatinina $(17,20-23)$.

- Albuminuria.

La proteinuria de peso molecular alto también ha sido referida como indicador no específico de exposición y, por tanto, tampoco es un indicador relevante $\left({ }^{16}\right)$.

- $\mathrm{N}$-acetil- $\beta$-D-glucosaminidasa (NAG).

H emos separado esta enzima, producto de secreción renal, porque es muy sensible para medir daño renal inducido por cadmio $\left({ }^{16,21}\right)$.

- Calcio urinario.

Si bien ya se había aceptado la cal ciuria como indicador de disfunción renal inducida por cadmio $\left({ }^{5}\right)$, es sólo recientemente que la escuela de Shangai ha postulado la excreción de calcio urinario como indicador temprano y específico de exposición ambiental. EI mecanismo por el que se produce la calciuria inducida por cadmio aún no ha sido bien determinado, pero se postula que el cadmio al ingresar a la célula podría causar cambios en la gradiente electroquímica por competición e inhibición en la bomba sodio-calcio, lo que afectaría la relación ciclo-adenosina monofosfato/ciclo-guanosinamonofosfato, que a su vez produciría disminución en la reabsorción del $\mathrm{Ca}^{2+}$ y por tanto su incremento en orina. Se sabe que la calciuria se correlaciona muy bien con incremento precoz de B2M y NAG, indicadores de disfunción tubular renal ${ }^{22}$ ).

El Tabla 1 resume las características toxicodinámicas del cadmio en el hombre.

- Perfil hepático

Útil para evaluar posible daño hepático en trabajadores de larga exposición, pues la alteración hepática aparece mucho después que el daño renal $\left({ }^{23}\right)$.
- Evaluación respiratoria:

Para evaluar el posible daño pulmonar, se usa radiografía de tórax y los principales indicadores de la función ventilatoria pulmonar:

- Capacidad vital forzada

- FEV $_{1}$

- $F E V_{1} / C V F$

- Pico de flujo espiratorio, etc (24).

Intoxicación crónica por exposición a cadmio:

En lo relativo a exposición/alteración-funciónrenal en expuestos a cadmio, se ha descrito el desarrollo de la intoxicación en tres fases:

1) En la primera fase, el cadmio que ingresa al organismo se acumula en la corteza renal y se liga a la metalotioneína. Si la ligazón cadmiometalotioneína no se satura, la eliminación de cadmio urinario está en relación directa a la cantidad acumulada en la corteza renal.

2) En exposiciones prolongadas, una segunda fase resulta en saturación de los sitios de unión cadmio-metalotioneína y, por tanto, el incremento del cadmio urinario en esta fase reflejará carga corporal.

3) U na tercera fase se caracteriza por disfunción renal, en la que excreción de cadmio está directamente relacionada al daño renal $\left({ }^{25,26}\right)$.

Vía de ingreso vs. manifestaciones clínicas y cáncer

Exceptuando los síntomas respiratorios, las manifestaciones clínicas son independientes de la vía de ingreso y del tipo de exposición. No hay estudios sobre si la ingestión del cadmio favorece el desarrollo del cáncer, pero sí que la exposición laboral podría estar asociada a incremento de riesgo de cáncer de próstata. Otros tipos de cáncer pulmonar o renal -hasta que no se confirme con nuevos estudios- deben ser considerados únicamente como probables, pues los datos actual es sólo sugieren riesgo bajo, aún con niveles de exposición elevados. Lo que sí se ha demostrado en animales es que el cadmio es teratógeno y embriotóxico $\left({ }^{27}\right)$. 
Tabla 1. Toxicología del cadmio.

Características toxi codi námicas en humanos.

\begin{tabular}{|c|c|}
\hline & \\
\hline Vida media en nasofaringe y traqueobronqui os & $<24 \mathrm{~h}$ \\
\hline Vida media en sangre & 2,5 meses \\
\hline Vida media en riñón & 4 a 60 años \\
\hline Vida media en hígado ..................... & 4 a 19 años \\
\hline Vida media en el organismo total & 30 a 40 años \\
\hline Promedio del período total de absorción .. & 14 días \\
\hline Por ciento absorción gastrointesti nal ..... & $5 \%$ \\
\hline
\end{tabular}

Concentraciones biológicas críticas:

- Corteza renal : $367 \mu \mathrm{g} / \mathrm{g}$ (Rango: 200 a 400)

- Riñón total : 2 a $20 \mathrm{mg}$

- Hígado: de 30 a $42 \mu \mathrm{g} / \mathrm{g}$

- Orina: $>5 \mu \mathrm{g} \mathrm{Cd} / \mathrm{g}$ de creatinina

BEIs y TLVs aceptados

BEIs:

- Cadmi o en orina: $\quad<5 \mu \mathrm{g} / \mathrm{g}$ creatinina.

- Cadmi o en sangre: $<5 \mu \mathrm{g} / \mathrm{L}$ de sangre total.

- Cadmio en la corteza renal : $<200 \mu \mathrm{g} / \mathrm{g}$ en peso húmedo.

- Calcio urinario: $2 \mu \mathrm{g} / \mathrm{g}$ creatinina

- B2M: <1500 $\mu \mathrm{g} / \mathrm{g}$ creatinina

- a GST: $<3,5 \mu \mathrm{g} / \mathrm{L}$

TLV:

- Cd ambiente de trabajo: 0,002 mg/ m³ para $8 \mathrm{~h} /$ día / 5 días semana

Compilado de KjellstromT and Nordberg G F ( $\left.{ }^{9}\right)$, Zenz C (6), Ellemhorn M J ( ${ }^{(4)}$ y ACGIH (19).

Síndromes de exposición crónica a cadmio

Para sistematizar el estudio de exposición crónica a cadmio, ambiental o laboral, se ha agrupado hallazgos de laboratorio, principalmente, que permiten describir los siguientes síndromes:

\section{Síndrome renal}

En exposiciones ocupacionales se ha encontrado que primero aparece disfunción glomerular y luego de un período de latencia, entre 10 y 20 años, recién se manifiesta la clásica microproteinuria $\left({ }^{27-29}\right)$.
El síndrome renal clásico de exposición a cadmio se caracteriza por:

- Proteinuria de peso molecular bajo, constituida principalmente por proteínas de tipo tubular, pero con predominio de proteínas especificas, como B2M, proteínas unidas al retinol, inmunoglobulinas de cadena corta 0 pos- $\omega$-proteínas, además de enzimas como lisozima, $\mathrm{N}$-acetil- $\beta$-D-glucosaminidasa, ribonucleasa y $\pi \mathrm{GST}(16,21,23,30)$.

- Aumento en la eliminación de la enzima lisososma- $\beta$-galactosidasa, lo que sugiere daño a nivel de algunas células epiteliales de vías urinarias ${ }^{21}$ ). 
- Calciuria: En exposición ocupacional o ambiental prolongada hay daño en la reabsorción tubular, Io que trae alteración del metabolismo del calcio con calciuria y aún formación de cálculos (22).

- Glucosuria, aminoaciduria y fosfaturia $\left({ }^{25}\right)$.

- Incremento de los niveles de urea, creatinina y ácido úrico en suero, por falla en el aclaramiento renal $\left({ }^{26}\right)$.

- Proteinuria de peso molecular alto de tipo glomerular, albúmina principalmente, pero también de transferrina e IgG $\left({ }^{27}\right)$.

\section{Síndrome de disfunción pulmonar}

En la exposición aguda se describe un síndrome de irritación de vías respiratorias y, en exposición crónica, síndromes obstructivos y restrictivos e inclusive fibrosis pulmonar. Sin embargo, en la evaluación clínica de alteración de la función pulmonar en expuestos es necesario puntualizar:

- Primero, es imprescindible valorar correctamente el hábito de fumar, pues humo de tabaco y cadmio potencian su acción sobre bronquios y pulmones.

- Segundo, estudios epidemiológicos señalan que la mortalidad por enfermedad respiratoria crónica es mayor en individuos con antecedentes de exposición que en no expuestos.

- Tercero, si se separa al trabajador del ambiente contaminado, no se detiene necesariamente la evolución de la enfermedad $(24,26)$.

\section{Síndrome óseo: itaí-itaí.}

U no de los primeros cuadros clínicos descritos atribuible exclusivamente a exposición ambiental fue el del cadmio y se le denominó "itaíitaí", cuya traducción al inglés sería "ouchouch" ergo, en español es "ay-ay", onomatopeya de las quejas debidas a los fuertes dolores que producía la osteomalacia entre los pobladores expuestos en la zona de Toyama (Japón), luego de la Segunda Guerra M undial, donde se le describió por primera vez en zonas agrícolas con altos índices de contaminación por $\mathrm{Cd}$ y $\mathrm{Zn}$ en el agua de los cultivos de arroz. Posteriormente se ha demostrado que la enfermedad ambiental ocurre principalmente en sujetos con metabolismo de hueso osteoporótico, como mujeres multíparas 0 , en ambos sexos, en personas sedentarias mayores de 50 años y también en algunos casos de trastornos del metabolismo del calcio o ingesta baja de proteínas y de vitamina D. En exámenes radiográficos de pacientes con "itaí-itaí" y en expuestos ocupacional es a cadmio se ha demostrado seudo-fracturas. Los primeros daños óseos descritos en expuestos que ingerían arroz contaminado era descalcificación y todavía sigue como el primer indicio de exposición que puede terminar en osteomalacia, deformaciones óseas manifiestas, fracturas espontáneas, lumbalgia, parestesias y neuralgias en miembros inferiores (14,26,28,29,31-34).

\section{Síndrome cardiovascular}

Se ha descrito hipertensión arterial, además de daño en la pared de las arterias, como las principales manifestaciones de la exposición ocupacional a cadmio. Sin embargo, estudios epidemiológicos en expuestos ambientales no han sido concluyentes para establecer causalidad entre exposición y efectos cardiovasculares $(8,12)$.

\section{Otras manifestaciones:}

Entre las manifestaciones generales no patognomónicas tenemos $\left({ }^{4}\right)$ :

- Anosmia, pérdida de peso, decaimiento general.

- Coloración amarilla de los dientes e incremento de frecuencia de caries dental.

- Síntomas gastointestinales variados.

- A nemia moderada, por alteración en el transporte del Fe dentro de las células eritropoyéticas, similar a la producida por el plomo.

- Leucocitosis y linfocitosis.

- Daño hepático moderado y, por tanto, disminución de la capacidad metabólica del híga- 
do en general y en particular para los xenobióticos.

- Atrofia testicular primaria en exposiciones ocupacionales a altos niveles de Cd.

U so de BEIs y TLVs para evaluación del riesgo cadmio

Revisemos ahora los parámetros de mayor uso en la actualidad para evaluar ocupacionalmente riesgo cadmio, con la salvedad de que en nuestro país sólo se usa $\mathrm{Cd}$ urinario expresado en $\mu \mathrm{g} / \mathrm{L}$ y muy ocasionalmente $B 2 \mathrm{M}$, aún en exposiciones altas, como en las grandes empresas metalúrgicas, agravado por que el BEI nacional es muy alto, $20 \mu \mathrm{g} / \mathrm{L}\left({ }^{35}\right)$. $Y$ aunque resulte una afirmación de perogrullo, todos los efectos agudos y crónicos del $\mathrm{C}$ d sobre riñón y sistema respiratorio pueden ser prevenidos manteniendo los TLV s (valores límites umbrales) y BEIs por debajo de sus niveles críticos, entendiéndose como tales los niveles mínimos del xenobiótico con los cuales no se tiene efectos adversos.

L a protección ambiental debería comenzar por hacer un inventario de las fuentes contaminadoras, principalmente las grandes industrias metalúrgicas extractivas de plomo y zinc, para recomendar se mantengan las emisiones bajo los niveles aceptados como "permisibles".

En cuanto al trabajador, su protección debería estar a cargo de médicos ocupacionistas, con la intervención activa del higienista industrial, pues no se concibe la acción del uno con el trabajador y del otro sobre el ambiente de labor. EI médico debe aplicar el criterio de los BEIs y de ellos cadmio urinario expresado en $\mu \mathrm{g}$ por gramo de creatinina y proteinuria de peso molecular bajo o NAG, a los que se les considera como los BEIs de mejor calidad para el adecuado pronóstico de carga corporal actual y de daño futuro a la salud del trabajador, respectivamente. El higienista industrial es responsable de mantener el nivel contaminación ambiental laboral dentro de valores TLV s determinado por los organismos normativos nacionales $y$, a falta de éstos, por los internacionales, como la
NIOSH, AGCIH, WHO u OIT, y debe recomendar aplicar tecnología de mejora del ambiente laboral para lograr un nivel adecuado del tóxico. Actualmente se considera que el TLV para $\mathrm{Cd}$ es $0,002 \mathrm{mg} / \mathrm{m}^{3}$ para partículas por debajo de $5 \mathrm{~mm}$ para 40 horas/semana de exposición, durante todo el período de la vida laboral $(19,36)$.

Por último, la recomendación actual de salud e higiene ocupacional es que se aplique medidas correctivas cuando el cadmio ambiental Ilegue a su nivel de acción, Ilamado no crítico, de $2 \mu \mathrm{g} / \mathrm{m}^{3}$ o cuando el trabajador alcance concentraciones de cadmio en orina $5 \mu \mathrm{g} / \mathrm{g}$ de creatinina 0 de $5 \mu \mathrm{g} / \mathrm{L}$ de sangre total $\left({ }^{37}\right)$.

Como conclusión, el cadmio es un xenobiótico ubicuo y de comprobada toxicidad para el hombre y los ecosistemas. Su alta permanencia en el medio ambiente y sus innumerables usos lo hacen un elemento de toxicidad similar o mayor que la del plomo para el medio ambiente y para el trabajador, con el agravante de ser poco conocido y estudiado.

Para afrontar el problema medioambiental y laboral, es necesario actualizar su conocimiento toxicológico y, como primera medida preventivo promocional en salud ambiental, se debería hacer un inventario de las fuentes industriales emisoras, para facilitar su fiscalización; y, en salud ocupacional, evaluar la metodología del examen a expuestos industriales para aplicar indicadores biológicos de exposición acordes con el estado actual del conocimiento.

\section{BIBLIOGRAFÍA}

1. La Dou J. Medicina Laboral y A mbiental. 2a. Edición. M éxico: Ed. El M anual M oderno. 1999.

2. Alarcón AC. Contaminación $M$ inero $M$ etalúrgica del Lago Chinchaycocha y del Rio M antaro. Instituto para el Desarrollo de la Pesca y la M inería. IPEM IN, La Oroya 1994.

3. Ramírez AV, Seminario OM, Silva JG. Impacto toxicológico producido por la Fundición La Oroya en Ios habitantes de la ciudad al edaña. Comunicación a la Gerencia Central de la EM del CP. Documento de trabajo. 1993.

4. L evy BS, Wegman DH, Eds. Occupational Health, Recognising and Preventing Work Related Diseases. $2^{\text {nd }}$ Edition. Boston, USA :Little, Brown and C 0. 1998. 
5. Ellis KJ, Vartsky D, Zanzi I, Cohn S, Y asumuru S. Cadmium: in vivo measurement in smokers and non smokers. Science 1979; 205: 323.

6. Kjellstrom T, Nordberg GF. Kinetic model of cadmium metabolism. En: Fridberg L, Elinder CG, Kellstrom T, Nordberg GF, Eds. Cadmium and Health, A Toxicological and Epidemiological A ppraisal. Vol I, Boca Raton, FL:CRC Press. 1985: 179-97.

7. González Fernández E. Toxicocinética y evaluación de riesgos para la salud producidos por exposición a cadmio. M edicina y Seguridad del Trabajo. 1988. XXXV: 3-17.

8. Piotrowski J K, Coleman DO. Environmental Hazards of Heavy M etals: Summary Evaluation of $\mathrm{Pb}, \mathrm{Cd}$ and $\mathrm{Hg}$ ". M arc Report № 20, M ARC, Chelsea College, U niversity of L ondon. London 1990.

9. K jellstrom T, Nordberg G F. A kinetic model of Cd metabolism in the human being. Environ Res 1978; 16: 248-69.

10. Oleru UG. Kidney, liver, hair and lungs as indicators of $\mathrm{Cd}$ absorption. Am Ind Hyg A ssoc J 1976; 37: 617-30.

11. Kido T, Shaikh ZA, K ito H, H onda R, Nogawa K. Doseresponse relationship between urinary cadmium and metallothionein in a Japanese population environmentally exposed to cadmium. Toxicology 1991; 65: 325-32.

12. Lauwerys RR. L'mpact sanitaire de la pollution par le cadmium en Belgique: Etat de la question. M émoires de I'A cadémie Royale de M edécine de Belgique 1986; 141: 453-9.

13. Vuori R, Huunan-Seppala A, K ilpio J O, Salmela SS. Biologically active metals in human tissues: II. The effect of age on the concentration of $\mathrm{Cd}$ in aorta, heart, kidney, liver, lung, pancreas and skeletal muscle. Scand J Work Environ Health 1979; 5: 16-48.

14. Ellemhorn MJ. Medical Toxicology. $2^{\text {nd }}$ Edition. Baltimore, USA:Williams \& Wilkins. 1996.

15. J in T, L effler P, Nordberg G F. Cadmium-metallothionein nephrotoxicity in the rat; transient calciuria and proteinuria. Toxicology 1987; 45: 307-17.

16. Kawada T, Koyama H, Suzuki S. Cadmium, NAG activity, and $\mathrm{B} 2 \mathrm{M}$ in the urine of cadmium pigment workers. $\mathrm{Br}$ J Ind M ed 1989; 46: 52-5.

17. Berggard I, Beran AG. I solation and properties of a low molecular weight B2M occurring in human biological fluids. J Biol Chem 1988; 643: 4095- 103.

18. Nordberg GF. (Ed): Effects and Dose- Response Relationships of Toxic metal. A msterdam:Elsevier. 1976.

19. A merican Conference of Governmental Industrial Hygienist. Threshold limit values and biologic exposure indices for 1997-1998. Cincinnati. U SA. 1997.

20. Travis CC, Haddock AG. Interpretation of the observed age-dependency of $\mathrm{Cd}$ body burdens in man. Environ Res 1980; 22: 46-52.
21. J in T, Nordberg GF, W u X, et al. U rinary N-acetyl-b$D$-glucosaminidase isozymes as biomarker of renal dysfunction caused by cadmium in a general population. Environ Res 1999; 81: 167-73.

22. Wu $X$, J in T, Wang $Z, Y$ e $T$, Kong $Q$, Nordberg G. U rinary calcium as a biomarker of renal dysfunction in a general population exposed to cadmium. J Occup Environ M ed 2001; 43: 898-904.

23. Campbell J AH. Immunohistology localization of alpha, mu and pi class of glutathione S-tranferase in human tissues. Cancer (Philadelphia) 1991; 67: 1608-13.

24. Ramirez AV. Cadmium pollution in La Oroya, Peru. PA HO Bulletin 1986; 20: 375-9.

25. Lerner S, Hong CD y Bozian RC. Cadmium nephropathy - A clinical evaluation. J Occup M ed 1979; 21: 409-21.

26. Zenz C, Ed. Occupational M edicine, $3^{\text {rd }}$ Edition St. L ouis, USA :Ed M osby - Year Book Inc. 1994.

27. Nordberg GF. Current issues in low-dose cadmium toxicology, nephrotoxicity and carcinogenicity. Environ Sci 1996; 133: 137-45.

28. Nernard A. The Detection of Early Nephrotoxic Effects: Development and Validation of Screening Test through Clinical, Epidemiological and Experimental Studies (Thesis). L ouvain, Belgium, Catholic University of L ouvain: 1998.

29. Buchet J P, Lauwerys R, Roels $\mathrm{H}$. Renal effects of cadmium body burden of the general population. Lancet 1990; 2: 699702.

30. J arup L, Elinder CG, Spang G. Cumulative blood-cadmium and tubular proteinuria: a dose-response relationship. Int A rch Occup Environ Health 1988; 60: 223-9.

31. Nordberg G F, J in T, Kong Q, et al. Biological monitoring of cadmium exposure and renal effects in a population group residing in a polluted area in China. Sci Total Environ 1997; 45: 111-4.

32. Frigerg $L$. Health hazards in the manufactures of alkaline accumulators with special reference to chronic cadmium poisoning. Act M ed Scand 1980; 138: 1-124.

33. Staessen J , Amery A, Bernard A, Bruaux P, Buchet J P. Effects of exposure to cadmium on calcium metabolism. A population study. Br J Ind M ed 1991; 48: 710-14.

34. Scott R, Haywood J K, Boddy K, Williams ED, H arvery $M$, Paterson $\mathrm{PJ}$. Whole body $\mathrm{Ca}$ deficit in $\mathrm{C}$ d exposed workers with hypercalciuria. U rology 1980; 15: 356-9.

35. M inisterio de Energía y M inas. Perú. DS- 046-2 001- EM Lima, Julio 20, 2001.

36. WHO. Environmental Health Criteria 134, Cadmium, Geneva:W HO. 1992.

37. J urup L, Berlund $M$, Elinder CG, Nordberg $G$, V ather $M$. Health effects of cadmium exposure-a review of the literature and a risk estimate. Scand J Work Environ H ealth 1999; 24: 21-34. 